\title{
TWO-TEMPERATURE INTRACLUSTER MEDIUM IN MERGING CLUSTERS OF GALAXIES
}

\author{
Motokazu TAKIZAWA \\ Department of Astronomy, Faculty of Science, Kyoto University, Sakyo-ku, Kyoto \\ 606-8502, JAPAN
}

\begin{abstract}
We investigate the evolution of intracluster medium during a cluster merger, explicitly considering the relaxation process between the ions and electrons by N-body and hydrodynamical simulations. When two subclusters collide each other, a bow shock is formed between the centers of two substructures and propagate in both directions along the collision axis. The shock primarily heats the ions because the kinetic energy of an ion entering the shock is larger than that of an electron by the ratio of masses. In the post-shock region the energy is transported from the ions to electrons via Coulomb coupling. However, since the energy exchange timescale depends both on the gas density and temperature, distribution of electron temperature becomes more complex than that of the plasma mean temperature, especially in the expanding phase. After the collision of two subclusters, gas outflow occurs not only along the collision axis but also in its perpendicular direction. The gas which is originally located in the central part of the subclusters moves both in the parallel and perpendicular directions. Since the equilibrium timescale of the gas along these directions is relatively short, temperature difference between ions and electrons is larger in the directions tilted by the angles of $\pm 45^{\circ}$ with respect to the collision axis. The electron temperature could be significantly lower that the plasma mean temperature by $\sim 50 \%$ at most. The significance of our results in the interpretation of X-ray observations is briefly discussed.
\end{abstract}

Subject headings: galaxies: clusters: general — hydrodynamics - intergalactic medium — plasmas — X-rays: galaxies

\section{INTRODUCTION}

Clusters of galaxies (CG) contain collisionless particles, galaxies and dark matter, and a diffuse gas component. The gas component is called intracluster medium (ICM). The 
ICM is the plasma with temperature of about $10^{8} \mathrm{~K}$, thus emitting X-ray mainly through the thermal bremsstrahlung of the electrons (Sarazin 1988). Since the ICM is optically thin for X-rays and spreads over the entire cluster, it can provide good information regarding the physical property of the clusters.

According to the hierarchical clustering scenario, it is believed that CG are formed through subcluster mergers and/or absorption of smaller galaxy groups. The rate of mergers are dependent on the cosmological parameters, especially on the density parameter, $\Omega_{0}$ (Richstone, Loeb, \& Turner 1992). However, when this is applied to more realistic situations such as observational results (eg., West \& Bothun 1990; Rhee et al. 1991; Jones \& Forman 1992; Mohr, Fabricant, \& Geller 1993; Bird 1994; Serna \& Gerbal 1996; Gurzadyan \& Mazure 1998; Solanes, Salvador-Soló, \& González-Casado 1998) or cosmological numerical simulations (Evrard et al. 1993; Jing et al. 1995; Crone, Evrard, \& Richstone, 1996), uncertainty of dynamical property of CG during mergers seriously affects the estimation of $\Omega_{0}$ (Nakamura, Hattori, \& Mineshige 1995).

To study evolution of CG, plenty of numerical simulations have been performed so far using N-body and hydrodynamical codes. Perrenod (1978) was the first to calculate the evolution of a spherical symmetric CG with a standard mesh hydrodynamical code. Recently, Takizawa\& Mineshige (1998) calculated the spherical CG and investigate the effect of pre-heating on ICM density profiles. To investigate the formation and evolution in more realistic situations, many simulations starting from the cosmological initial density perturbations are performed (e.g., Evrard 1990; Katz \& White 1993; Metzler \& Evrard 1994; Navarro, Frenk, \& White 1995; Bryan \& Norman 1998; Eke, Navarro, \& Frenk 1998; Yoshikawa, Itoh, \& Suto 1998). Schindler \& Müller (1993) find that characteristic temperature structures occur in merging clusters through shock heating and adiabatic compression and expansion. On the other hand, there are simulations starting from rather idealized initial conditions to study the mergers in detail. Roettiger, Loken, \& Burns (1997) study general behaviors of head on collisions with a high resolution finite-difference code. Their observational consequences are discussed in more detail in Roettiger, Burns, \& Loken (1996). Ishizaka (1997) find that the specific energy ratio of ICM and galaxies, $\beta_{\text {spec }}$, is a good indicator of merging clusters and that it can be used to determine the phase of the merger. In particular, off-center collisions are investigated by Ricker (1998).

In recent years, rather complex electron temperature structures are revealed in merging CG through X-ray observations (e.g., Fujita et al. 1996; Honda et al. 1996; Churazov et al. 1998; Donnelly et al. 1998a; Donnelly et al. 1998b; Davis \& White 1998; Markevitch, Sarazin, \& Vikhlinin 1998). In addition, Hanami et al. (1998) find that the energy of iron $\mathrm{K} \alpha$ line is higher than that expected from the temperature in ionization equilibrium 
plasma in SC1329-313 in Shapley concentration. These results implicate there exits strong bulk flow motion in ICM of merging clusters. Temperature maps of ICM contain important information of mergers as well as $\beta_{\text {spec }}$ does. Some numerical simulations are tried to explain these observational results; Roettiger, Burns, \& Pinkney (1995) tried the modeling for A2256, and Ishizaka \& Mineshige (1996) for Coma. More detailed "anatomy" is done for A754 by Roettiger, Stone, \& Mushotzky (1998).

As mentioned above, temperature structures of ICM provide us with very important information about the phase of the mergers. However, we can find only the electron temperature through X-ray observations until now. In all the previous studies the discussion is based on the assumption that observed electron temperature $\left(T_{\mathrm{e}}\right)$ is the same as the plasma mean temperature $(\bar{T})$. However, we believe this assumption is problematic. The shock primarily heats the ions because the kinetic energy of an ion entering the shock is larger than that of an electron by the ratio of masses. In addition, the equilibrium timescale between electrons and ions $\left(t_{\text {eq }}\right)$ in typical CG becomes $10^{8} \sim 10^{9} \mathrm{yr}$ if Coulomb coupling is considered as the relaxation process (see Spitzer 1962):

$$
t_{\mathrm{eq}}=2.0 \times 10^{8} \mathrm{yr}\left(\frac{\ln \Lambda}{40}\right)^{-1}\left(\frac{n_{\mathrm{i}}}{10^{-3} \mathrm{~cm}^{-3}}\right)^{-1}\left(\frac{T_{\mathrm{e}}}{10^{8} \mathrm{~K}}\right)^{3 / 2},
$$

where $n_{\mathrm{i}}$ is the ion density, $T_{\mathrm{e}}$ is the electron temperature, and $\ln \Lambda$ is the Coulomb logarithm. Indeed, some CG have larger electron temperature gradients than those expected in numerical simulations (Markevitch 1996). A two-temperature model of ICM is one of solutions to solve this discrepancy (Fox \& Loeb 1997; Chiéze, Alimi, \& Teyssier, 1998; Ettori\&Fabian 1998; Takizawa 1998). In cluster mergers, it is believed that shock heating plays an important role in the evolution of ICM. Furthermore, according to the previous studies about mergers, timescale surviving complex temperature structures is an order of $10^{9} \mathrm{yr}$, which is comparable to $t_{\mathrm{eq}}$. Therefore, difference between $T_{\mathrm{e}}$ and $\bar{T}$ should be considered properly to study the evolution of $T_{\mathrm{e}}$ structure in mergers.

To investigate the evolution of two-temperature ICM in cluster mergers, we perform N-body and hydrodynamical simulations considering the relaxation process between ions and electrons. For the hydrodynamical part, we choose the smoothed particle hydrodynamics (SPH) method. Although SPH codes are not better for problems with shocks than mesh codes, its Lagrangean nature is suitable to deal the electron-ion coupling simply.

The rest of this paper is organized as follows. In $\$$ 国 we estimate analytically some physical quantities relevant to cluster mergers. In $\S$ 逐 we describe the adopted numerical methods and initial conditions for our simulations. In $\S$ 国 we present the results. In $\oint$ 国 we summarize the results and discuss their implications. 


\section{BASIC CONSIDERATIONS OF EQUILIBRIUM TIMES}

In this section, we consider the scaling laws of CG and the initial conditions of cluster mergers. We assume that the power spectrum of the cosmological density fluctuation field, $P(k)$, obeys power law, $P(k) \propto k^{n}$. According to the spherical model for the non-linear collapse (see Peebles 1980, Padmanabhan 1993, etc.), the virial radius $(r)$, the density $(\rho)$, and the virial temperature $(T)$ of the dark halo obey the scaling laws as follows,

$$
\begin{gathered}
r \propto M^{(n+5) / 6}, \\
\rho \propto M^{-(n+3) / 2}, \\
T \propto M^{(1-n) / 6},
\end{gathered}
$$

where $M$ is the total mass of the halo. The effective index of $P(k)$ of CDM is $-1 \sim-2$ in the galactic and CG scale. We consider the case of $n=-2$. Then the above relations are,

$$
\begin{gathered}
r \propto M^{1 / 2}, \\
\rho \propto M^{-1 / 2} \\
T \propto M^{1 / 2}
\end{gathered}
$$

Using these relations and the gas fraction, $f_{\mathrm{g}}$, we obtain the scaling laws of equilibrium timescale between ions and electrons, $t_{\mathrm{eq}} \propto\left(f_{\mathrm{g}} \rho\right)^{-1} T^{3 / 2}$, as follows.

$$
t_{\text {eq }} \propto f_{\mathrm{g}}^{-1} M^{5 / 4}
$$

Thus, the more massive CG is, the longer $t_{\text {eq }}$ is, if $f_{\mathrm{g}}$ is constant. However, the observational results about the luminosity-temperature (LT) relation suggests $f_{\mathrm{g}}$ depends on $M$ (Mushotzky 1984; Edge \& Stewart 1991; David et al. 1993; Fukazawa 1997; Mushotzky \& Scharf 1997; Markevitch 1998; Arnaud \& Evrard 1998). Since $L \propto\left(f_{\mathrm{g}} \rho\right)^{2} T^{1 / 2} R^{3}, f_{\mathrm{g}} \propto M^{3 / 8}$ if we assume $L \propto T^{3}$. Then we obtain

$$
t_{\mathrm{eq}} \propto M^{7 / 8}
$$

$t_{\text {eq }}$ is longer in more massive CG also in this case, though the dependence is slightly weaker than the case when $f_{g}$ is constant.

Next, let us consider the the merger of subclusters whose masses are $M_{1}$ and $M_{2}$, respectively. The collision velocity $V$ is estimated as follows. From the dynamical energy conservation law, we obtain,

$$
-\frac{G M_{1} M_{2}}{2 R}=-\frac{G M_{1} M_{2}}{r_{1}+r_{2}}+\frac{1}{2} M V^{2}
$$


where $G$ is the gravitational constant, $r_{1}$ and $r_{2}$ is the virial radius of the each subcluster, $R$ is the maximum expansion radius of the whole system, and $M=M_{1} M_{2} /\left(M_{1}+M_{2}\right)$ is the reduced mass. Since the maximum expansion radius is twice the virial radius, using the relation (5) we obtain

$$
R=2 \times\left(\frac{M_{1}+M_{2}}{M_{1}}\right)^{1 / 2} r_{1}
$$

It is convenient to introduce the parameter $\alpha \equiv M_{2} / M_{1}$. Without losing the generality, we can set $0<\alpha<1$. Then, from the scaling relations of (5) and equations (10) and (11), we can describe $V$ as follows,

$$
V^{2}=\frac{2 G M_{1}}{r_{1}}(1+\alpha)\left[\frac{1}{1+\alpha^{1 / 2}}-\frac{1}{4(1+\alpha)^{1 / 2}}\right]
$$

On the other hand, the sound speed of subcluster $1, c_{1}$ is

$$
c_{1}^{2}=\gamma(\gamma-1) \frac{G M_{1}}{2 r_{1}},
$$

where $\gamma$ is the ratio of specific heat. Thus, the Mach number, $\mathcal{M}_{1}=V / c_{1}$, is,

$$
\mathcal{M}_{1}^{2}=\frac{4(1+\alpha)}{\gamma(\gamma-1)}\left[\frac{1}{1+\alpha^{1 / 2}}-\frac{1}{4(1+\alpha)^{1 / 2}}\right] \text {. }
$$

From the scaling relation (5), the Mach number for subcluster $2, \mathcal{M}_{2}$, is,

$$
\mathcal{M}_{2}^{2}=\alpha^{-1 / 2} \mathcal{M}_{1}^{2}
$$

Figure [1 shows $\mathcal{M}_{1}^{2}$ (solid line) and $\mathcal{M}_{2}^{2}$ (dotted line) with respect to $\alpha$ when $\gamma=5 / 3$. For major mergers $(\alpha>0.1)$, these values are greater than $\sim 2$. Thus, in CDM universe, supersonic collisions are quite natural in major mergers.

The Mach number gives the maximum temperature difference between ions and electrons in the post-shock regions. The specific inertial energy of ions and electrons are, in the limit of no mutual energy exchange,

$$
\begin{aligned}
& \frac{k_{\mathrm{B}} T_{\mathrm{i}}}{\mu m_{\mathrm{p}}} \sim c^{2}+V^{2}, \\
& \frac{k_{\mathrm{B}} T_{\mathrm{e}}}{\mu m_{\mathrm{p}}} \sim c^{2},
\end{aligned}
$$

where $\mu$ is the mean molecular weight, $m_{\mathrm{p}}$ is the proton mass, $k_{\mathrm{B}}$ is the Boltzmann constant, and $c$ is the sound speed in the pre-shock regions. Thus the difference between $T_{\mathrm{e}}$ and 
$\bar{T} \sim\left(T_{\mathrm{i}}+T_{\mathrm{e}}\right) / 2$, is

$$
\frac{T_{\mathrm{e}}}{\bar{T}} \sim \frac{1}{1+\mathcal{M}^{2} / 2}
$$

Thus, from figure 1, we expect $T_{\mathrm{e}} / \bar{T}$ is less than about 0.6 . We will confirm this later by numerical simulations.

\section{THE SIMULATIONS}

In the present study, we consider the CG consisting of two components: collisionless particles corresponding to galaxies and DM and two-temperature gas corresponding to the ICM. When calculating gravity, both components are considered, although the former dominates over the latter. Radiative cooling and heat conduction are not included in our simulations.

\subsection{The Numerical Method}

To solve the hydrodynamical equations for the gas component, we used the smoothedparticle hydrodynamics (SPH) method (see Monaghan 1992). Although more accurate finite-difference methods better handle shocks than SPH methods, its fully Lagrangean nature is convenient to calculate the evolution of two-temperature plasmas since the coupling between ions and electrons can be treated more simply in Lagrangean view as follows.

As the standard SPH codes for one-temperature fluid, we solve the continuity equation, the momentum equation, and the thermal energy equation with artificial viscosity to treat the shocks. In addition to these equations, we solve one more equation for the normalized electron temperature, $\tilde{T}_{\mathrm{e}} \equiv T_{\mathrm{e}} / \bar{T}$, where $T_{\mathrm{e}}$ is the electron temperature and $\bar{T}$ is the plasma mean temperature. We assume that artificial viscous heating is effective only for ions and that only the Coulomb coupling is considered in the relaxation process. Then, the Lagrangean time evolution of $\tilde{T}_{\mathrm{e}}$ is (see Appendix),

$$
\frac{d \tilde{T}_{\mathrm{e}}}{d t}=\frac{\tilde{T}_{\mathrm{i}}-\tilde{T}_{\mathrm{e}}}{t_{\mathrm{eq}}}-\tilde{T}_{\mathrm{e}} \frac{Q_{\mathrm{vis}}}{u},
$$

where $\tilde{T}_{\mathrm{i}} \equiv T_{\mathrm{i}} / \bar{T}$ is the normalized ion temperature, $Q_{\text {vis }}$ is the artificial viscous heating per unit mass, and $u$ is the thermal energy per unit mass. For the numerical integration 
of equation (20), we should take a time-step shorter enough than not only the Courant and viscous timescale as usual SPH codes (see Monaghan 1992 §10.3) but also $t_{\mathrm{eq}}$. In our simulations, however, the latter is much shorter than the former in the central high-density regions. Thus, the integration with timestep relevant to $t_{\text {eq }}$ takes a huge computational time. In addition, since two-temperature nature is not important in such regions, the time-step control above mentioned is not a good choice. To integrate equation (20) with the Courant and the viscous time-step control, we use the results of Fox \& Loeb (1997) as follows. Let us integrate equation (20) from $t=t_{0}$ to $t=t_{0}+\Delta t$. First, we integrate equation (20) regarding the second term on the right-hand side being negligibly small. In this case, neglecting the small change of Coulomb logarithm in $t_{\mathrm{eq}}$, we can integrate this analytically (Fox \& Loeb 1997),

$$
\frac{\Delta t}{t_{2 \mathrm{~s}}}=\frac{n_{\mathrm{i}}}{n_{\mathrm{i}}+n_{\mathrm{e}}}\left[F\left(\tilde{T}_{\mathrm{e}}\left(t_{0}+\Delta t\right)\right)-F\left(\tilde{T}_{\mathrm{e}}\left(t_{0}\right)\right)\right],
$$

where,

$$
F(x)=\ln \left(\frac{1+\sqrt{x}}{1-\sqrt{x}}\right)-2 \sqrt{x}\left(1+\frac{x}{3}\right),
$$

and $t_{2 \mathrm{~s}} \equiv t_{\mathrm{eq}}\left(t_{0}\right) \tilde{T}_{e}\left(t_{0}\right)^{-3 / 2}$. Second, we add the contribution from the second term to $\tilde{T}_{\mathrm{e}}\left(t_{0}+\Delta t\right)$ using the second-order Runge-Kutta method. If the time step is chosen correctly, the displacement of the position of each SPH particle is smaller enough than the spatial resolution and $Q_{\text {vis }} / u$ is smaller enough than unity. Thus the above method can follow the evolution of $\tilde{T}_{\mathrm{e}}$ efficiently and with reasonable accuracy.

Gravitational forces are calculated by the Barnes-Hut tree algorithm (Barnes \& Hut 1986) and softened using a Plummer potential profile $\Phi \propto\left(r^{2}+\epsilon^{2}\right)^{-1}$, where $\epsilon$ is the softening parameter. We set $\epsilon$ one tenth of the initial core radius of the smaller subcluster in the simulation. Tree structure is also used to search for nearest neighbors in SPH calculations (Hernquist \& Katz 1989). In the standard one-dimensional shock tube test, our code nicely reproduced the analytic solution. The shock and contact discontinuity is broadened over a range of about three times of smoothing length. During the calculations described below, the total energy is conserved within $\sim 0.4 \% /(1000$ steps $)$.

\subsection{Models and Initial Conditions}

We consider mergers of two virialized subclusters of galaxies with masses $M_{1}$ and $M_{2}$. Initial configuration of each subcluster is described as follows. The spatial distribution of 
the DM in each subcluster is represented by the King distribution;

$$
\rho_{\mathrm{DM}}(r)= \begin{cases}\rho_{0}\left[1+\left(r / r_{\mathrm{c}}\right)^{2}\right]^{-3 / 2} & \left(r \leq r_{\text {out }}\right) \\ 0 & \left(r>r_{\text {out }}\right)\end{cases}
$$

where, $\rho_{0}$ is the central density, $r_{\mathrm{c}}$ is the core radius, and $r_{\text {out }}$ is the radius of the cluster. We set $r_{\text {out }}=5 \times r_{\mathrm{c}}$. This is nearly equal to the virial radius of the King distribution. $\rho_{0}$ is determined by the total mass of DM $\left(M_{\mathrm{DM}}\right)$,

$$
\rho_{0}=\frac{M_{\mathrm{DM}}}{4 \pi r_{\mathrm{c}}^{3}}\left[-\frac{t_{\mathrm{out}}}{\sqrt{t_{\mathrm{out}}^{2}+1}}+\ln \left|t_{\mathrm{out}}+\sqrt{t_{\mathrm{out}}^{2}+1}\right|\right]^{-1},
$$

where $t_{\text {out }}=r_{\text {out }} / r_{\mathrm{c}}$. We assume velocity distribution of the DM particles to be an isotropic Maxwellian. Then, from the second moment of the collisionless Boltzmann equation, one-dimensional velocity dispersion at $r, \sigma_{1}^{2}(r)$, is (e.g., Binney \& Tremaine 1987)

$$
\sigma_{1}^{2}(r)=\frac{1}{\rho_{\mathrm{DM}}(r)} \int_{r}^{r_{\mathrm{out}}} \rho_{\mathrm{DM}}(r) \frac{G M_{r}}{r^{2}} d r
$$

where $M_{r}$ is the mass inside $r$. We assume that initial ICM temperature is isothermal and equal to the virial temperature at $r_{\text {out }}$. The ICM is initially in hydrostatic equilibrium within a cluster potential of the DM and the ICM, itself.

We set the initial conditions as follows. Two subclusters are placed at rest with a separation between the center of each subcluster, $r_{\text {sep }}$, initially. The coordinate system is taken in such a way that the center of masses is at rest in the origin. Two representative cases are examined in the preset study: a collision between two equal-mass clusters (Run A), an absorption of a smaller cluster by a larger one (Run B). The scaling law between the two subclusters and $r_{\text {sep }}$ are determined as the case of $P(k) \propto k^{-2}$ in $\$ 2$. In Run A, two subcluster have the same masses; $M_{1}=M_{2}=0.5 \times 10^{15} M_{\odot}$. We set the core radii to be $r_{\mathrm{c}}=0.2 \mathrm{Mpc}$. Each subcluster consists of 5000 collisionless particles and 5000 $\mathrm{SPH}$ particles. The total gas mass fraction is $10 \%$ for both clusters. In Run B, the mass ratio is $M_{1}: M_{2}=4: 1$. The larger cluster has the same mass as that in Run $\mathrm{A}$. The particle numbers of the smaller one are one fourth of the larger one. The parameters in our calculations are summarized in table 1.

\section{RESULTS}

\subsection{Time evolution of the total feature during the mergers}

Figure 2 shows the time evolution of various physical quantities representing the total feature of the system of Run A: from the top, the total luminosity $(L)$, the 
emissivity-weighted mean electron temperature $\left(T_{\mathrm{e}, \mathrm{ew}}\right)$, the square root of the velocity dispersion parallel to the collision axis $\left(\sigma_{/ /}\right)$, and the specific energy ratio of DM and ICM $\left(\beta_{\text {spec }}\right) . \beta_{\text {spec }}$ is defined as,

$$
\beta_{\text {spec }} \equiv \frac{\mu m_{\mathrm{p}} \sigma_{/ /}^{2}}{k_{\mathrm{B}} T_{\mathrm{e}, \mathrm{ew}}} .
$$

When calculating $L$, we assume the thermal bremsstrahlung of optically thin plasma (Rybicki \& Lightman 1979) and neglect the line emissions.

Around the collision, all of $L, T_{\mathrm{e}, \mathrm{ew}}$ and $\sigma_{/ /}$rise but on different timescales. Since the rise of $T_{\mathrm{e}, \mathrm{ew}}$ is quicker than that of $\sigma_{/ /}, \beta_{\mathrm{spec}}$ has two peaks in the time evolution. The minimum of $\beta_{\mathrm{spec}}$ is coincident with the maximum of $T_{\mathrm{e}, \mathrm{ew}}$.

Similar results are seen also in Run B (figure 7). However, the amplitudes of time fluctuation are smaller than in Run A.

Since the emissivity is proportional to the square of the gas density, emissivity-weighted mean temperature (EWMT) is more like the temperature in the central high density regions, where $t_{\mathrm{eq}}$ is $\sim 10^{8} \mathrm{yr}$ or less. Thus, the difference in EWMTs between electrons and ions is practically negligible. Indeed, this difference becomes $\sim 1 \%$ even at maximum in both runs. Therefore, our results agree qualitatively with those of the previous one-temperature simulations such as Ishizaka (1997).

\subsection{Time evolution of temperature distribution of Run A}

As seen in \$4.1, two-temperature nature of ICM does not manifest itself in EWMT of CG. This is not the case, however, when we can resolve the spatial distribution of ICM temperature. Let us examine first the case of Run A.

First of all, let us see the $\bar{T}$ map and the gas velocity field, and discuss the dynamical evolution of Run A. Figure 3 shows the snapshots of X-ray surface brightness (contours) and emissivity-weighted $\bar{T}$ (colors) distribution seen from the direction perpendicular to the collision axis. X-ray surface brightness contours are equally spaced on a logarithmic scale and separated by a factor of 7.4. The blue, green, yellow, and red colors correspond to $k_{\mathrm{B}} T \sim 5 \mathrm{keV}, 10 \mathrm{keV}, 15 \mathrm{keV}$, and $20 \mathrm{keV}$, respectively. The times are listed above each panel: $t=4.1,4.3,4.6$, and $4.85 \mathrm{Gyr}$ correspond to the first maximum of $\beta_{\mathrm{spec}}$, the minimum of $\beta_{\mathrm{spec}}$, the second maximum of $\beta_{\mathrm{spec}}$, and the minimum of $T_{\mathrm{e}, \mathrm{ew}}$, respectively. Figure 1 shows the evolution of the gas velocity field, seen from the same direction at the same times as in figure 3. The longest vector corresponds to the maximum velocity listed below each panel. 
When two subclusters just contact each other at $t=3.5$ Gyr (upper left panel), $\bar{T}$ rises slightly at the interface between the two subclusters due to the adiabatic compression but we can clearly distinguish two individual clusters through the X-ray image. Then the two approach each other and we see double peaks in the X-ray emissivity profile of 'one cluster' at $t=4.1$ Gyr (upper middle). Just between the peaks $\bar{T}$ rises up to $\sim 20 \mathrm{keV}$ due to a shock. The shock front is perpendicular to the collision axis. However, since $\bar{T}$ still remains nearly the initial value $(\sim 5 \mathrm{keV})$ around the peaks of the X-ray image, EWMT does not increase so much that $\beta_{\text {spec }}$ becomes rather large (figure 21). At the most contracting phase ( $t=4.3 \mathrm{Gyr}$ : upper right), two peaks merge to one peak in the X-ray image and the X-ray image elongates to the directions perpendicular to the collision axis. Since the high temperature $(\sim 20 \mathrm{keV})$ region is located around the X-ray peak, EWMT becomes maximum (figure 2). Then the cluster expands and two shocks propagate in the opposite directions along the collision axis $(t=4.6 \mathrm{Gyr}$ : lower left). We can clearly see two 'lens-shaped' high temperature regions associated to the shocks in the $\bar{T}$ structure. We can also see the cooler region $(\sim 5 \mathrm{keV})$ spread in the directions perpendicular to the collision axis. These characteristic temperature structures reflect the fact that the gas outflow occurs

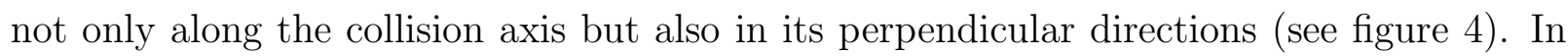
these directions the gas is effectively cooled by the adiabatic expansion and emits more X-ray than the hottest regions. Thus, EWMT decreases, though high temperature regions still exist. At the most expanding phase $(t=4.85$ Gyr: lower middle), the X-ray image elongates along the collision axis. The shocks reach in very low density region. We see an 'X-shaped' region with slightly high temperature (indicated by light blue color). This structure is also due to adiabatic expansion associated to the gas outflows. Then the system contracts again and settles down to the spherical structure ( $t=7$ Gyr: lower right). Owing to the subsonic accretion of the gas along the collision axis, slightly high temperature regions are created on the both sides of the collision axis (see figure (1).

Note that there can be complex temperature structures in ICM when X-ray images do not have definite substructures. It is certain that image elongation is occurred in these case, but this is severely affected by the viewing angles. Thus, temperature map is more suitable for the diagnostic of merging clusters.

Next, let us see the $T_{\mathrm{e}}$ map and discuss the difference between $\bar{T}$ and $T_{\mathrm{e}}$. Figure 5 shows the same as figure 3 , but for $T_{\mathrm{e}}$. Although in the contracting phase $(t \leq 4.3 \mathrm{Gyr}) T_{\mathrm{e}}$ maps are similar to the $\bar{T}$ maps, in the expanding phase $(t>4.3 \mathrm{Gyr})$ both maps are rather different. To see the difference between $\bar{T}$ and $T_{\mathrm{e}}$ in detail, we make the snapshots of the distribution of $\tilde{T}_{\mathrm{e}} \equiv T_{\mathrm{e}} / \bar{T}$. Figure 6 shows the $\tilde{T}_{\mathrm{e}}$ maps of Run A. The red, yellow, green, and blue colors correspond to $\tilde{T}_{\mathrm{e}} \sim 0.1,0.3,0.5$, and 0.7 , respectively. 
In the contracting phase $(t \leq 4.3 \mathrm{Gyr})$, the difference between $\bar{T}$ and $T_{\mathrm{e}}$ is rather small. At $t=4.1 \mathrm{Gyr}, T_{\mathrm{e}}$ is slightly lower than $\bar{T}$ at the shock. In overall there are no significant two-temperature regions. Then, at $t=4.3 \mathrm{Gyr}$, split two-temperature regions appear due to the propagation of shocks. Since $t_{\mathrm{eq}}$ is longer in the outer parts where density is low, two-temperature regions are more spread there than in the central parts. In this phase, however, two-temperature nature is less important than in the later phase because the the shocks is nearly standing shock and $t_{\text {eq }}$ is shorter in the central parts.

On the other hand, in the expansion phase (4.3 Gyr $\leq t \leq 4.85 \mathrm{Gyr})$, the distributions of $\bar{T}$ and $T_{e}$ are clearly qualitatively different. From figure 6 we find two common features both at $t=4.6 \mathrm{Gyr}$ and at $t=4.85 \mathrm{Gyr}$. One is that $T_{e}$ is significantly lower $(\sim 50 \%)$ than $\bar{T}$ in the post-shock high $\bar{T}$ regions. These regions are spread on $\sim 0.5 \mathrm{Mpc}$ scale behind the shocks. The other is that the difference between $\bar{T}$ and $T_{\mathrm{e}}$ is larger in the directions tilted by the angles of $\pm 45^{\circ}$ with respect to the collision axis. This is also due to the gas outflows both along the collision axis and in its perpendicular directions. The gas originally located in the central part, whose $t_{\text {eq }}$ is relatively short, moves outward in these directions. Note that $t_{\mathrm{eq}}$ is hardly changed by the adiabatic compression and expansion when $\gamma=5 / 3$. Thus, the difference between $\bar{T}$ and $T_{\mathrm{e}}$ is less in these directions. As a result, we see four peaks in the $T_{\mathrm{e}}$ map at $t=4.6 \mathrm{Gyr}$ and the 'X-shaped' high temperature region becomes less contrasted in the $T_{\mathrm{e}}$ map at $t=4.85 \mathrm{Gyr}$. Note that high temperature regions around $\sim 20 \mathrm{keV}$ cannot be seen in the $T_{\mathrm{e}}$ maps of the expanding phase. Therefore, it is probable that we underestimate the collision velocity by $\sim 30 \%$ if we estimate it from the hottest regions in $T_{\mathrm{e}}$ map in the expanding phase. Two-temperature nature still remains at $t=7$ Gyr in the outer parts as the case of spherical models (Fox \& Loeb 1997; Takizawa 1998).

\subsection{Time evolution of temperature distribution of Run B}

First, we describe the dynamical evolution of Run B through the $\bar{T}$ map and the gas velocity field. Figure 8 shows the same as figure 3 , but for Run B. Temperature color scale is adjusted for Run B. The blue, green, yellow, and red colors correspond to $k_{\mathrm{B}} T \sim 4 \mathrm{keV}$, $7 \mathrm{keV}, 10 \mathrm{keV}$, and $13 \mathrm{keV}$, respectively. The times are listed above each panel: $t=3.9$, $4.1,4.3$, and 4.75 Gyr correspond to the first maximum of $\beta_{\mathrm{spec}}$, the minimum of $\beta_{\mathrm{spec}}$, the second maximum of $\beta_{\mathrm{spec}}$, and the minimum of $T_{\mathrm{e}, \mathrm{ew}}$, respectively. Figure 9 shows the same as figure 1 , but for Run B.

When the two subclusters approach each other, the bow shock with an arc shape is formed just between the two. Thus, the arc-shaped hot region is seen between the two peaks of the X-ray image at $t=3.9$ Gyr (upper middle). This region clearly separates 
the component of larger subcluster $(\sim 5 \mathrm{keV})$ from the component of smaller subcluster $(\sim 2.5 \mathrm{keV})$. Then, the two peaks merge to one triangle image of X-ray $(t=4.1 \mathrm{Gyr}$ : upper right). The hottest temperature region $(\sim 13 \mathrm{keV})$ is located in the peak of the $\mathrm{X}$-ray image and the hot region associated to the bow shock is seen elongated slantingly backward with respect to the motion of the smaller subclusters. Then the gas expands and the two shocks propagate outward along the collision axis. The one in front of the motion of the smaller subcluster (for the left of the figure) is arc-shaped and the other is rather flat $(t=4.3$ Gyr: lower left $)$. At the most expanding phase $(t=4.75$ Gyr: lower middle) the X-ray image elongates along the collision axis and the shocks reach very low density regions. The elongation of the image is more significant for the left of the figure. Then the system contracts again and settles down to the spherical structure ( $t=7$ Gyr: lower right). Accretion flows are seen only from the front side with respect to the motion of the smaller subcluster.

Next, we describe two-temperature nature of Run B through the $T_{\mathrm{e}}$ map and the $\tilde{T}_{\mathrm{e}}$ map. Figure 10 shows the same as figure 8, but for $T_{\mathrm{e}}$ and figure 11 shows the same as figure 6, but for Run B. As the case of Run A, in the contracting phase $(t \leq 4.1 \mathrm{Gyr})$ the

$T_{\mathrm{e}}$ distribution is similar to the $\bar{T}$ one though $T_{\mathrm{e}}$ is slightly lower than $\bar{T}$ around the shock. On the other hand, in the expanding phase $(t>4.1 \mathrm{Gyr})$ substantial deviation emerges especially around the hot regions associated to the shocks. At $t=4.3 \mathrm{Gyr}$ in the $\bar{T}$ map both two hot regions have almost the same temperature $(\sim 13 \mathrm{keV})$, whereas in the $T_{\mathrm{e}}$ map, the former (with respect to the motion of the smaller subcluster) hot region becomes less than $\sim 10 \mathrm{keV}$. On the other hand, in the backward hot region, $\sim 13 \mathrm{keV}$ component still remains. In this region, $t_{\mathrm{eq}}$ is rather short because the gas there originates from the smaller subcluster (see the scaling relation (8)). At $t=4.75 \mathrm{Gyr}$, as the case of Run A, $\tilde{T}_{\mathrm{e}}$ is low in the directions tilted by the angles of $\pm 45^{\circ}$ with respect to the collision axis, although this tendency is less significant than in Run A.

\section{SUMMARY AND DISCUSSION}

We investigate evolution of ICM during mergers considering the relaxation process between the ions and electrons. From the simple analytical estimation, we find that in the $\mathrm{CDM}$ universe the equilibrium timescale between ions and electrons are longer in more massive CG even if the gas fraction is dependent on CG mass in such a way that the X-ray luminosity is proportional to the cube of the temperature. We estimate the collision velocity and show that supersonic collisions are quite natural in CDM universe in major mergers and that electron temperature can be less than a half of the plasma mean temperature in 
the post-shock regions. Temperature estimated from X-ray observations can be significantly lower than ion temperature.

We carry out the numerical simulations of the mergers by N-body and hydrodynamical simulations, incorporating the relaxation process between the ions and electrons. To solve the evolution of the normalized electron temperature, we adopt the results of Fox \& Loeb (1997). The difference in emissivity-weighted mean temperatures of ions and electrons is practically negligible during the merger; the discrepancy is $\sim 1 \%$ even at maximum. On the other hand, the spatial distribution of the electron temperature is significantly different from that of the plasma mean one especially in low-density regions in the expanding phase. In this phase, electron temperature is at most $\sim 50 \%$ lower than the plasma mean one in the post-shock hot regions. In addition, the difference between them is more enhanced in the directions tilted by the angles of $\pm 45^{\circ}$ with respect to the collision axis. When the two subclusters have different masses, the hot region located to the former position (with respect to the direction of the motion of the smaller subcluster) has lower electron temperature than that located backward in the expanding phase.

Recently, Markevitch et al. (1998) estimate the subcluster collision velocity of Cygnus A using the electron temperature map obtained by ASCA. From this temperature map and the ROSAT PSPC image, it is likely that Cygnus A is in just contracting phase such as Run A at $t=4.1$ Gyr. Thus, two-temperature nature is almost negligible in this case. However, collision velocities are likely underestimated by $\sim 30 \%$ if we use the electron temperature of the hottest regions of merging clusters in the expanding phase such as SC1329-313 (Hanami et al. 1998).

Although we consider only head-on collisions for simplicity in the present calculations , off-center collisions should be investigated to model more realistic situations. Ricker (1998) find that that spiral bow shocks occur in off-center mergers, which probably produce rather complex electron temperature distribution. We will investigate this issue as a future work.

We consider only the classical Coulomb coupling as the relaxation process between ions and electrons. It is possible, however, in ICM more efficient relaxation processes could be effective (McKee \& Cowie 1977; Pistinner, Levinson, \& Eichler 1996). In this case the equilibrium timescale could be shorter than the value given by equation (11). Therefore, the temperature difference between ions and electrons can be less than our results. If magnetic field exits in ICM, it is possible that electrons are also significantly heated by shocks associated with MHD instabilities. Also in this case the temperature difference between ions and electrons could be less.

We neglect the heating process from the galaxies to ICM. If substantial amount of 
thermalized hot gas is injected into ICM from the galaxies, temperature difference could be less. It is possible that mergers of $\mathrm{CG}$ activate the star formation in the member galaxies through the galaxy-galaxy interaction, galaxy-ICM interaction, etc (Caldwell et al. 1993) . In this case, this effect cannot be negligible but the effect on ICM temperature distribution is rather sensitive on the detailed modeling of the star formation activity (Fujita 1998).

We adopt the SPH method for the hydrodynamical part in the present simulations for simplicity to treat the coupling between ions and electrons. However, resolution of the shock in SPH is not better than that in high resolution finite-difference codes such as TVD, PPM, CIP, etc, because artificial viscosity is used to treat the shocks in SPH. Furthermore, bulk viscosity is included in our code and possibly effects electron temperature slightly. Although we believe that our simulations can follow the qualitative behavior, it is useful to simulate two-temperature ICM during mergers with finite-difference codes.

The author would like to thank C. Ishizaka, H. Hanami, and S. Mineshige for helpful comments. This work is supported in part by a Grant-in-Aid from the Ministry of Education, Science, Sports and Culture of Japan (6179) and Research Fellowships of the Japan Society for the Promotion of Science for Young Scientists.

\section{A. DERIVATION OF THE EQUATION FOR THE NORMALIZED ELECTRON TEMPERATURE}

In the SPH calculations shocks are treated through artificial viscosity. We assume that artificial viscous heating is effective only for ions and that only Coulomb coupling is considered in the relaxation process. Then the Laglangean time evolution of the electron temperature $T_{\mathrm{e}}$ and the mean temperature $\bar{T}=\left(n_{\mathrm{e}} T_{\mathrm{e}}+n_{\mathrm{i}} T_{\mathrm{i}}\right) /\left(n_{\mathrm{e}}+n_{\mathrm{i}}\right)$ is

$$
\begin{aligned}
\frac{d T_{\mathrm{e}}}{d t} & =(\gamma-1) \frac{T_{\mathrm{e}}}{n} \frac{d n}{d t}+\frac{T_{\mathrm{i}}-T_{\mathrm{e}}}{t_{\mathrm{eq}}} \\
\frac{d \bar{T}}{d t} & =(\gamma-1) \frac{T}{n} \frac{d n}{d t}+\frac{2}{3} \frac{\mu m_{\mathrm{p}}}{k_{\mathrm{B}}} Q_{\mathrm{vis}}
\end{aligned}
$$

where $T_{\mathrm{i}}$ is the ion temperature, $n$ is the gas density, and $\gamma=5 / 3$ is the ratio of specific heats and $Q_{\text {vis }}$ is the artificial viscous heating per unit mass. We use the artificial viscosity in the form described in Monaghan (1992) §4.1, which is the most commonly used one. Then, the explicit expression of $Q_{\text {vis }}$ for the $i$-th SPH particle is

$$
Q_{\mathrm{vis}, i}=\frac{1}{2} \sum_{j} m_{j} \Pi_{i j}\left(\boldsymbol{v}_{i}-\boldsymbol{v}_{j}\right) \cdot \nabla_{i} W_{i j}
$$


where $\Pi_{i j}$ is given by

$$
\Pi_{i j}= \begin{cases}\frac{-\alpha \bar{c}_{i j} \mu_{i j}+\beta \mu_{i j}^{2}}{\bar{\rho}_{i j}}, & \text { for }\left(\boldsymbol{v}_{i}-\boldsymbol{v}_{j}\right) \cdot\left(\boldsymbol{r}_{i}-\boldsymbol{r}_{j}\right)<0 \\ 0, & \text { for }\left(\boldsymbol{v}_{i}-\boldsymbol{v}_{j}\right) \cdot\left(\boldsymbol{r}_{i}-\boldsymbol{r}_{j}\right)>0\end{cases}
$$

and

$$
\mu_{i j}=\frac{h_{i j}\left(\boldsymbol{v}_{i}-\boldsymbol{v}_{j}\right) \cdot\left(\boldsymbol{r}_{i}-\boldsymbol{r}_{j}\right)}{\left(\boldsymbol{r}_{i}-\boldsymbol{r}_{j}\right)^{2}+\eta^{2}}
$$

In the above expressions, $m_{j}, \boldsymbol{v}_{j}$, and $\boldsymbol{r}_{j}$ are the mass, velocity, and position of the $j$-th SPH particle, respectively. $c_{i j}, \rho_{i j}$ and $h_{i j}$ are the average of sound speed, density, and smoothing length of particle $i$ and $j$, respectively. We set $\alpha=1, \beta=2$, and $\eta^{2}=0.01$, which are typical values in SPH calculations. $\nabla_{i} W_{i j}$ is the gradient of the $j$-th particle's kernel function at $\boldsymbol{r}_{i}$.

Introducing the temperature normalized by $\bar{T}$, i.e., $\tilde{T}_{\mathrm{e}} \equiv\left(T_{\mathrm{e}} / \bar{T}\right)$ and $\tilde{T}_{\mathrm{i}} \equiv\left(T_{\mathrm{i}} / \bar{T}\right)$, we find

$$
\frac{d \tilde{T}_{\mathrm{e}}}{d t}=\frac{\tilde{T}_{\mathrm{i}}-\tilde{T}_{\mathrm{e}}}{t_{\mathrm{eq}}}-\tilde{T}_{\mathrm{e}} \frac{Q_{\mathrm{vis}}}{u}
$$

where $u \equiv 3 k_{\mathrm{B}} \bar{T} /\left(2 \mu m_{\mathrm{p}}\right)$ is the thermal energy per unit mass. 


\begin{tabular}{ccc}
\hline \hline & Run A & Run B \\
\hline$M_{1} / M_{2}\left(10^{15} M_{\odot}\right)$ & $0.5 / 0.5$ & $0.5 / 0.125$ \\
$r_{\mathrm{c}, 1} / r_{\mathrm{c}, 2}(\mathrm{Mpc})$ & $0.2 / 0.2$ & $0.2 / 0.1$ \\
$k_{\mathrm{B}} T_{1} / k_{\mathrm{B}} T_{2}(\mathrm{keV})$ & $4.78 / 4.78$ & $4.78 / 2.39$ \\
$r_{\mathrm{sep}}(\mathrm{Mpc})$ & 4.0 & 3.3 \\
$f_{\mathrm{g}}$ & 0.1 & 0.1 \\
$\epsilon(\mathrm{Mpc})$ & 0.02 & 0.01 \\
$N_{1} / N_{2}(\mathrm{SPH})$ & $5000 / 5000$ & $5000 / 1250$ \\
$N_{1} / N_{2}(\mathrm{DM})$ & $5000 / 5000$ & $5000 / 1250$ \\
\hline
\end{tabular}

Table 1: Model parameters 


\section{REFERENCES}

Arnaud, M., \& Evrard, A. E. 1998, submitted to MNRAS (astro-ph/9806353)

Barnes, J., \& Hut, P. 1986, Nature, 324, 446

Binney, J., \& Tremaine, S. 1987, Galactic Dynamics (Princeton: Princeton University Press)

Bird, C. M. 1994, AJ, 107, 1637

Bryan, G. L., \& Norman, M. L., 1998, ApJ, 495, 80

Caldwell, N., Rose, J. A., Sarples, R. M., Ellis, R. S., Bower R. G. 1993, AJ, 106, 473

Chiéze, J.-P., Alimi, J.-M, \& Teyssier, R. 1998, ApJ, 495, 630

Churazov, E., Gilfanov, M., Forman, W., \& Jones, C. 1998, ApJ, submitted astroph/9802166)

Crone, M. M., Evrard, A. E., \& Richstone, D. O. 1996, ApJ, 467, 459

David, L., Slyz, A., Jones, C., Forman, W., Vrtilek, S. D., \& Arnaud, K. A. 1993, ApJ, 412, 479

Davis, D. S. \& White III, R. E. 1998, ApJ, 492, 57

Donnelly, R. H., Markevitch, M., Forman, W., Jones, C., David, L. P., Churazov, E., \& Gilfanov, M. 1998a, ApJ, 500, 138

Donnelly, R. H., Markevitch, M., Forman, W., Jones, C., Churazov, E., \& Gilfanov, M. 1998b, ApJ, in press astro-ph/9809380

Edge, A. C., \& Stewart, G. C. 1991, MNRAS, 252, 414

Eke, V. R., Navarro, J. F., \& Frenk, C. S. 1998, ApJ, 503, 569

Ettori, S. \& Fabian, A. C. 1998, MNRAS, 293, L33

Evrard, A. E. 1990, ApJ, 363, 349

Evrard, A. E., Mohr, J. J., Fabricant, D. G., \& Geller, M. J. 1993, ApJ, 419, L9

Fox, D. C., \& Loeb, A. 1997, ApJ, 491, 459

Fujita, Y., Koyama, K., Tsuru, T., \& Matsumoto, H. 1996, PASJ, 48, 191

Fujita, Y. 1998, ApJ, 509, 587

Fukazawa, Y. 1997, PhD thesis, University of Tokyo

Gurzadyan, V. G.,\& Mazure, A. 1998, MNRAS, 295, 177

Hanami, H., Tsuru, T., Shimasaku, K., Yamauchi, S., Ikebe, Y., \& Koyama, K. 1998, ApJ, submitted 
Hernquist, L., \& Katz, N. 1989, ApJS, 70, 419

Honda, H., Hirayama, M., Watanabe, M., Kunieda, H., Tawara, Y., Yamashita, K., Ohashi, T., Hughs, J., P., \& Henry, J. P. 1996, ApJ, 473, L71

Ishizaka, C., \& Mineshige, S. 1996, PASJ, 48, L37

Ishizaka, C., 1997 Ap \& SpS, 254, 233

Jones, C., \& Forman, W. 1992, in Clusters and Superclusters of Galaxies, ed. Fabian, A. C. (Dordrecht: Kluwer), 49

Jing, Y. P., Mo, H. J., Borner, G., \& Fang, L. Z. 1995, MNRAS, 276, 417

Katz, N., \& White, S. D. M. 1993, ApJ, 412, 455

McKee, C. F., \& Cowie, L., L. 1977, ApJ, 215, 213

Markevitch, M. 1996, ApJ, 465, L1

Markevitch, M. 1998, ApJ, 504, 27

Markevitch, M., Sarazin, C. L., \& Vikhlinin, A. 1998, ApJ, submitted (astro-ph/9812005)

Metzler, C. A., \& Evrard, A. E. 1994, ApJ, 437, 564

Mohr, J. J., Fabricant, D. G., Geller, M. 1993, ApJ, 413, 492

Monaghan, J. J. 1992, ARA\&A, 30, 543

Mushotzky, R. F. 1984, Phys. Scripta, T7, 157

Mushotzky, R. F., \& Scharf, C. A. 1997, ApJ, 482, L13

Nakamura, F. E., Hattori, M., \& Mineshige, S. 1995, A\&A, 302, 649

Navarro, J. F., Frenk, C. S., \& White, S. D. M. 1995, MNRAS, 275, 720

Padmanabhan, T., 1993, Structure Formation in the Universe (Cambridge: Cambridge University Press)

Peebles, P. J. E., 1980, The Large-Scale Structure of the Universe (Princeton: Princeton University Press)

Perrenod, S. C. 1978, ApJ, 226, 566

Pistinner, S., Levinson, A., \& Eichler, D. 1996, ApJ, 467, 162

Rhee, G. F. R. N., van Harlem, M. P., Katgert. P., 1991, A\&A, 246,301

Richstone, D., Loeb, A., \& Turner, E. L. 1992, ApJ, 393, 477

Ricker, P., M. 1998, ApJ, 496, 670

Roettiger, K., Burns, J. O., \& Pinkney, J. 1995, ApJ, 453, 634 
Roettiger, K., Burns, J. O., \& Loken, C. 1996, ApJ, 473, 651

Roettiger, K., Loken, C., \& Burns, J. B. 1997, ApJS, 109, 307

Roettiger, K., Stone, J. M., \& Mushotzky, R. F. 1998, ApJ, 493, 62

Sarazin, C. L. 1988, X-ray Emission from Clusters of Galaxies (Cambridge: Cambridge University Press)

Schindler, S., \& Müller, E. 1993, A\&A, 272, 137

Serna, A.,\& Gerbal, D. 1996, A\&A, 309, 65

Solanes, J. M., Salvador-Soló, E., \& González-Casado, G. 1998, A\&A, in press (astro$\mathrm{ph} / 9812103)$

Spitzer, L., Jr. 1962, Physics of Fully Ionized Gases (New York: Wiley)

Takizawa, M., \& Mineshige, S. 1998, ApJ, 499, 82

Takizawa, M. 1998, ApJ, 509, 579

West, M. J., Bothun, G. D. 1990, ApJ, 350,36

Yoshikawa, K., Itoh, M., \& Suto, Y. 1998, PASJ, 50, 203 
Fig. 1. - The square of Mach numbers of two virialized clusters as functions of $\alpha$ (the ratio of the two cluster masses) during the merger for the case with $P(k) \propto k^{-2}$. The solid and dotted lines show the values for the larger and the smaller cluster, respectively.

Fig. 2.- The time evolution of various physical quantities representing the features of the entire system for Run A: from the top, (a) the total luminosity $(L)$, (b) the emissivityweighted mean electron temperature $\left(T_{\mathrm{e}, \mathrm{ew}}\right)$, (c) the square root of the velocity dispersion parallel to the collision axis $\left(\sigma_{/ /}\right)$, and $(d)$ the specific energy ratio of DM and ICM $\left(\beta_{\text {spec }}\right)$.

Fig. 3.- The snapshots of X-ray surface brightness (contours) overlaid with emissivityweighted $\bar{T}$ (colors) distribution seen from the direction perpendicular to the collision axis for Run A. X-ray surface brightness contours are equally spaced on a logarithmic scale and separated by a factor of 7.4. The blue, green, yellow, and red colors correspond to $k_{\mathrm{B}} T \sim 5$ $\mathrm{keV}, 10 \mathrm{keV}, 15 \mathrm{keV}$, and $20 \mathrm{keV}$, respectively. The times are listed above each panel.

Fig. 4. - The evolution of the gas velocity field for Run A seen from the same direction at the same times as in figure 3. The longest vector corresponds to the maximum velocity listed below each panel.

Fig. 5.- Same as figure 3, but for $T_{\mathrm{e}}$ instead of $\bar{T}$ for Run A

Fig. 6. - The snapshots of distribution of normalized electron temperature, $\tilde{T}_{\mathrm{e}} \equiv T_{\mathrm{e}} / \bar{T}$ for Run A. The red, yellow, green, and blue colors correspond to $\tilde{T}_{\mathrm{e}} \sim 0.1,0.3,0.5$, and 0.7 , respectively.

Fig. 7.- Same as figure 2, but for Run B.

Fig. 8.- Same as figure 3, but for Run B. Temperature color scale is adjusted for Run B. The blue, green, yellow, and red colors correspond to $k_{\mathrm{B}} T \sim 4 \mathrm{keV}, 7 \mathrm{keV}, 10 \mathrm{keV}$, and 13 $\mathrm{keV}$, respectively.

Fig. 9.- Same as figure 4 , but for Run B.

Fig. 10.- Same as figure 5, but for Run B.

Fig. 11. - Same as figure 6, but for Run B. 


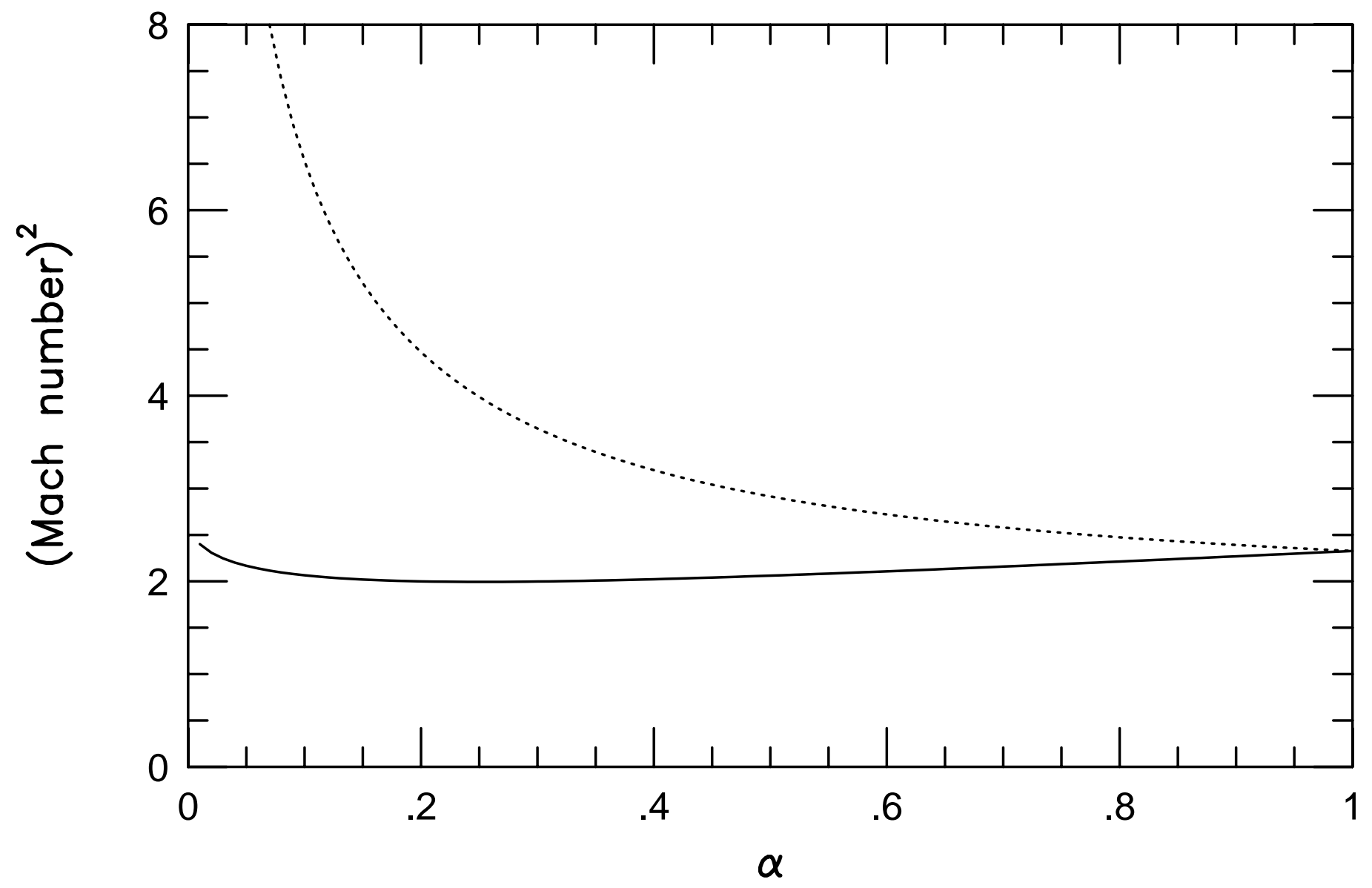



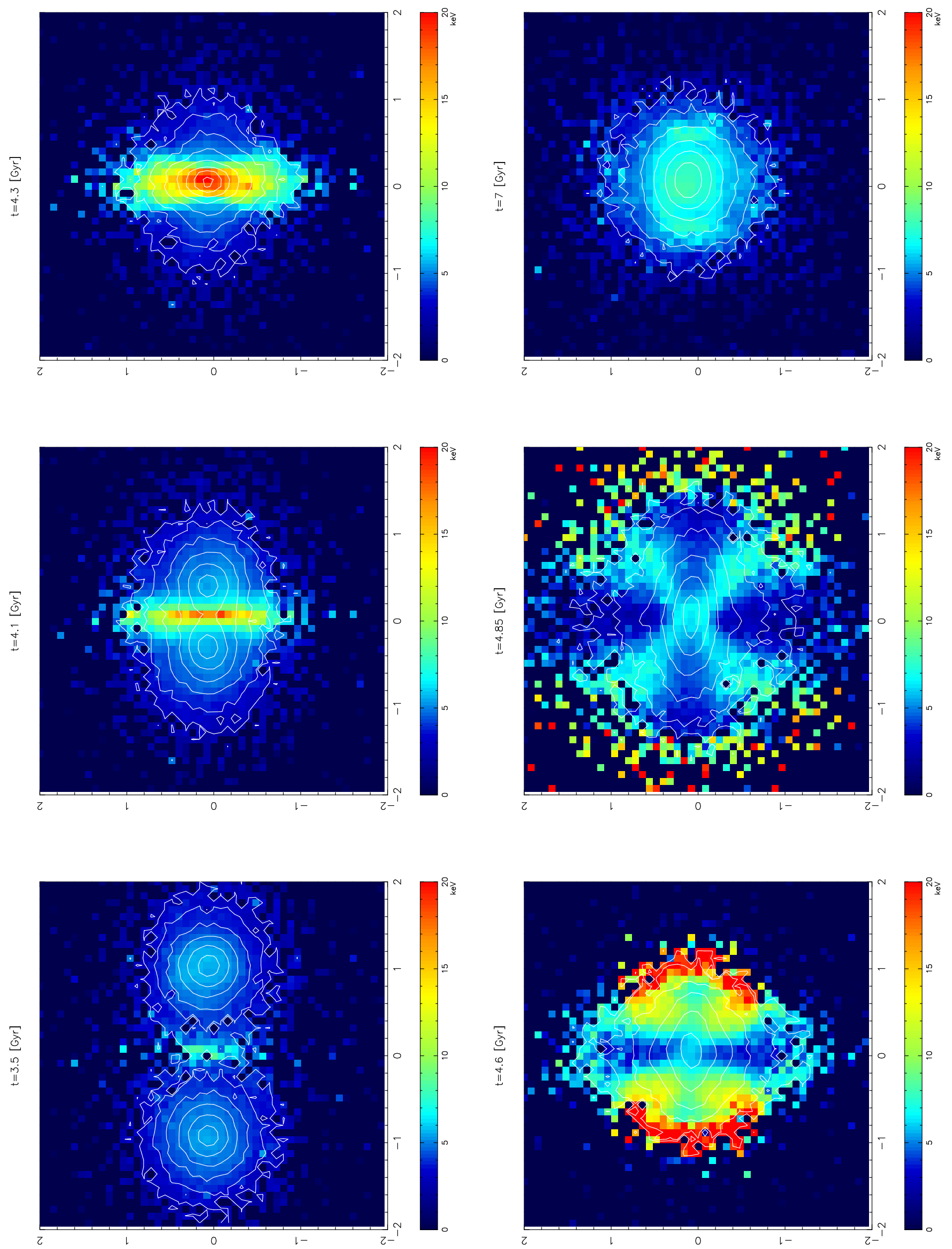

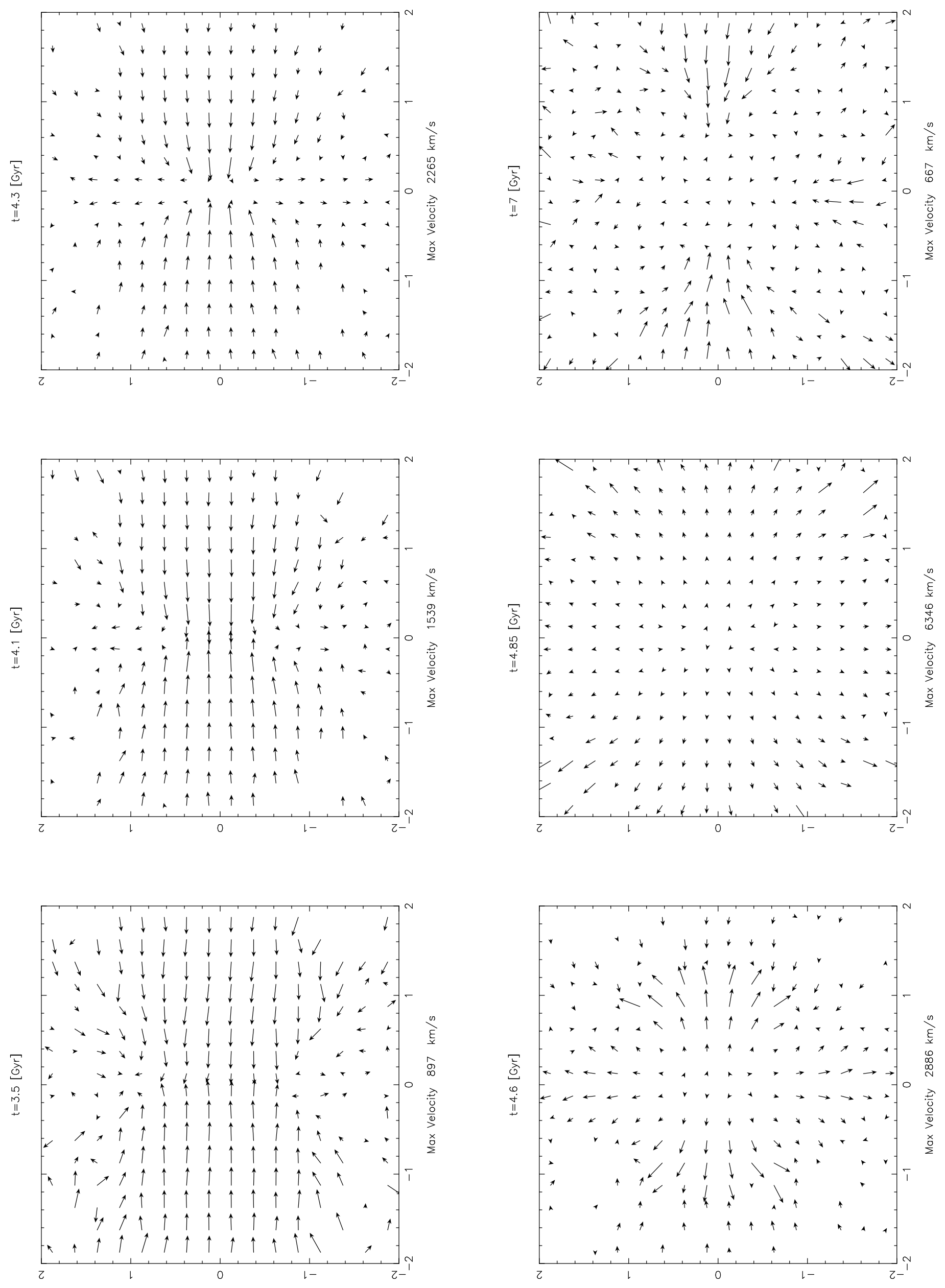

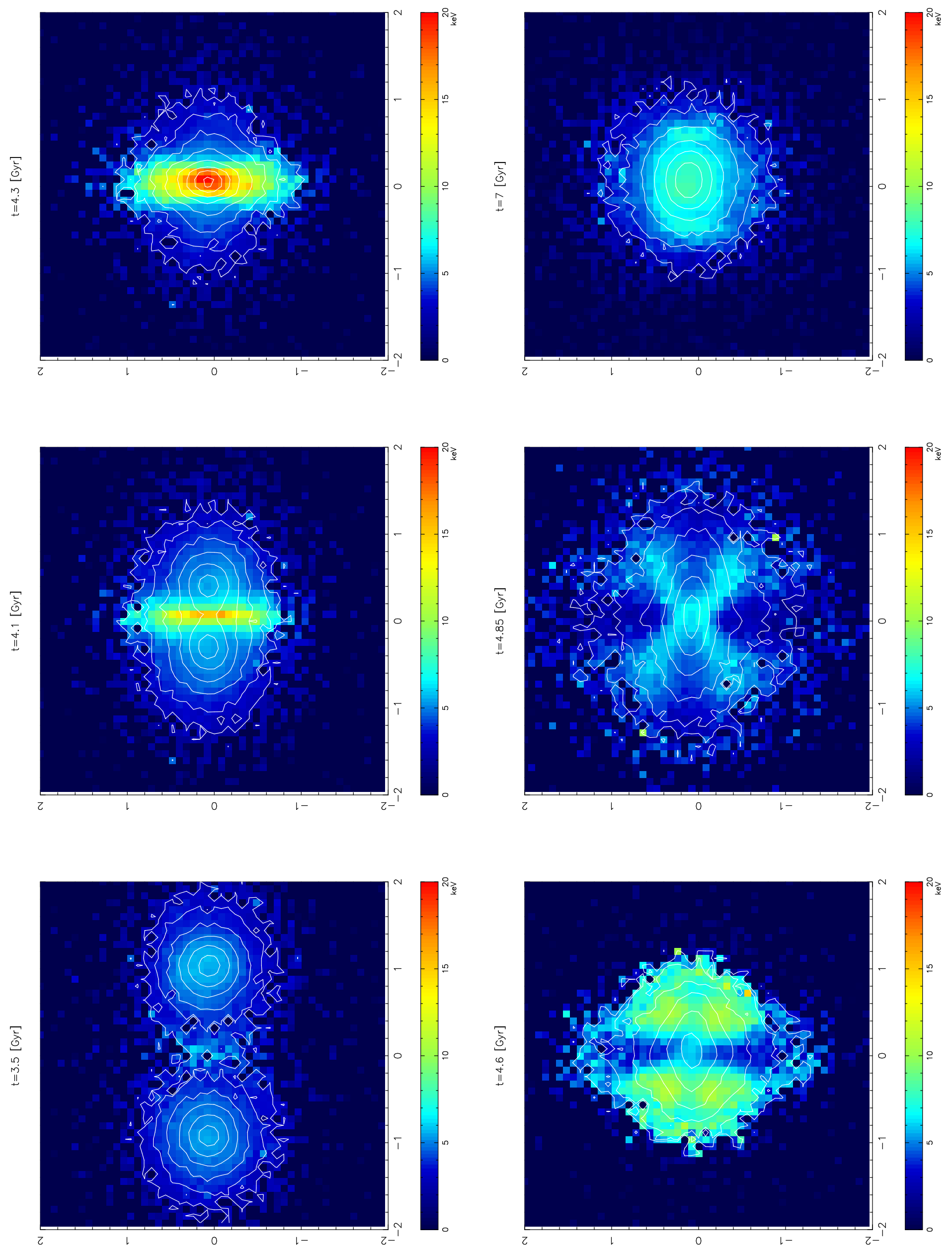

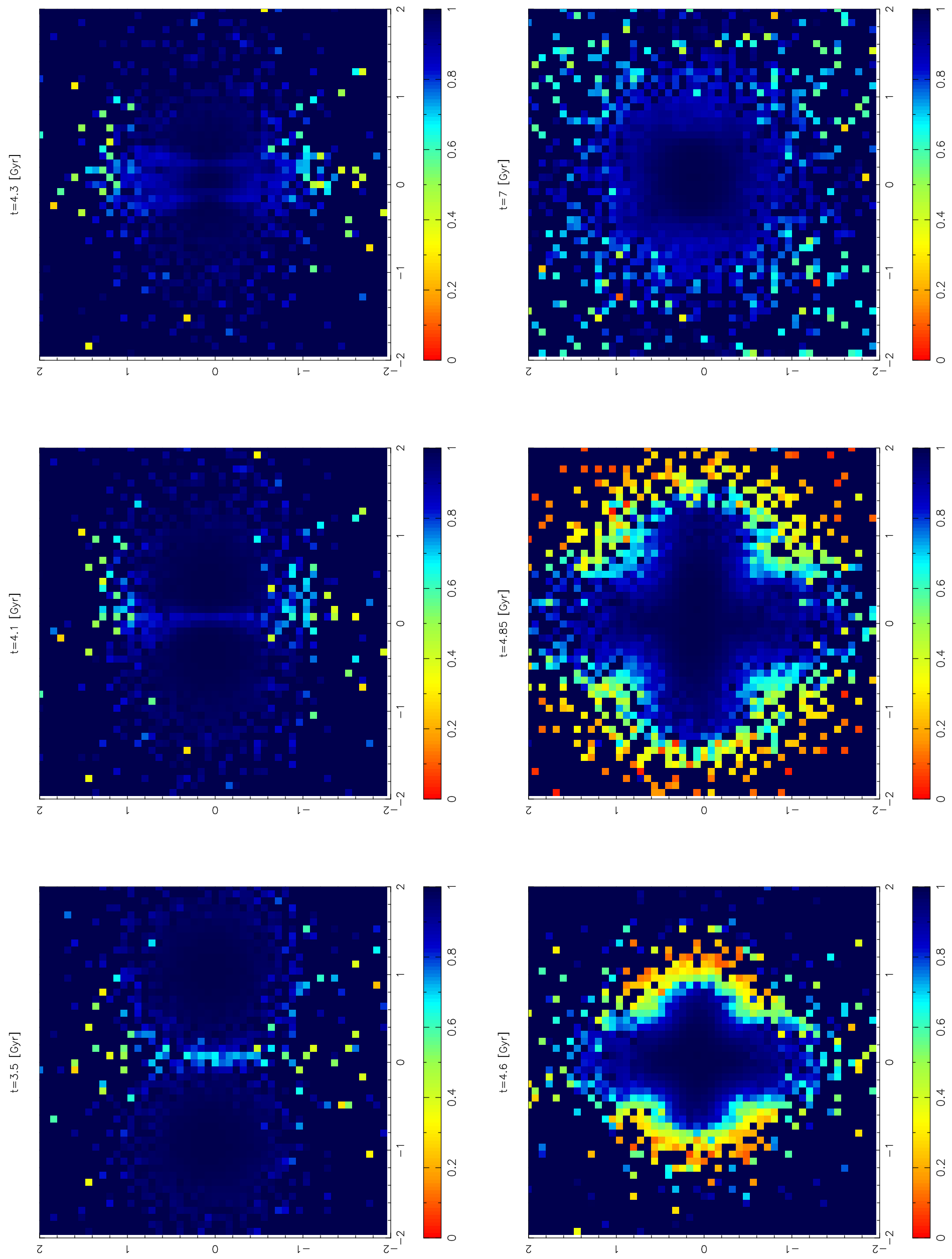

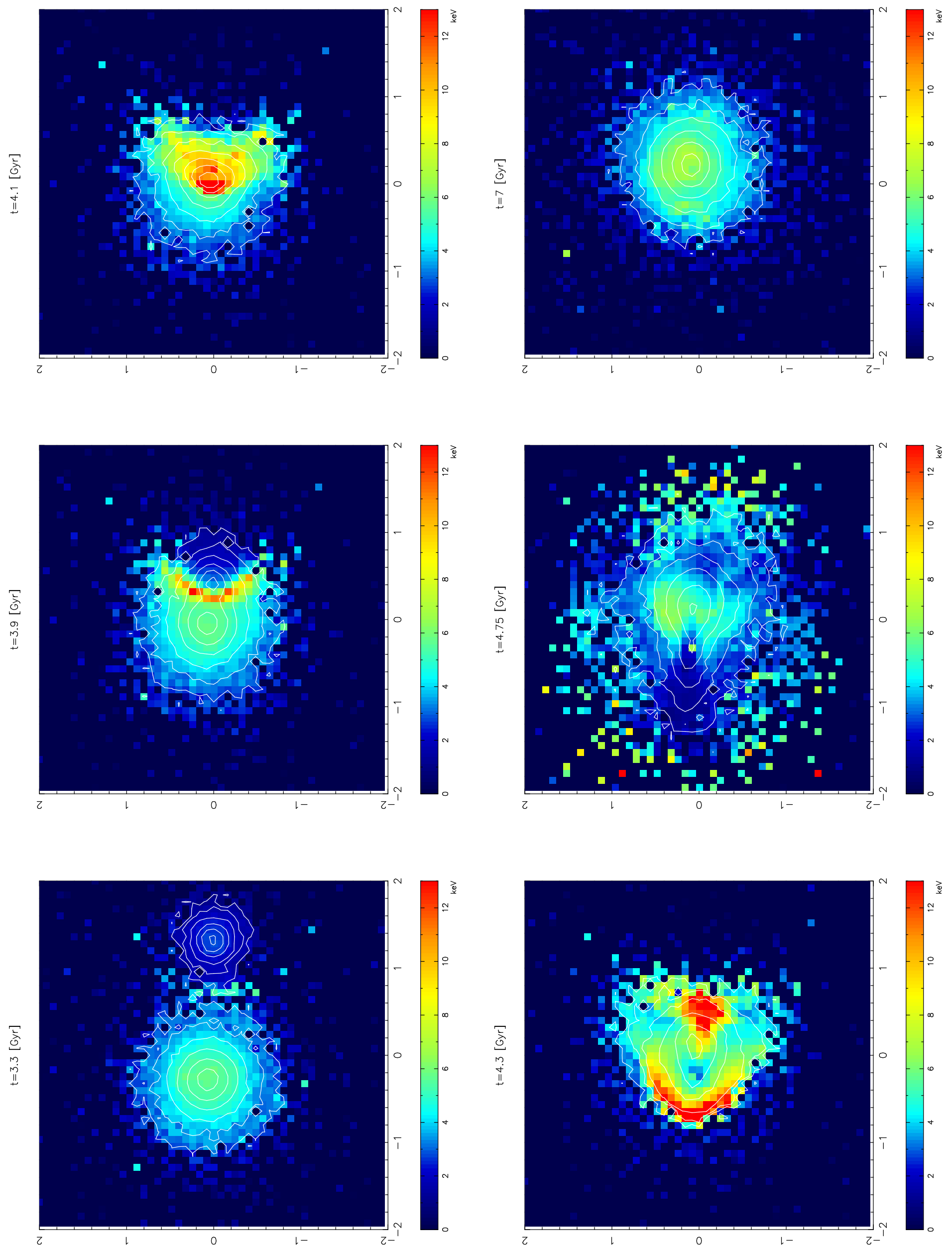

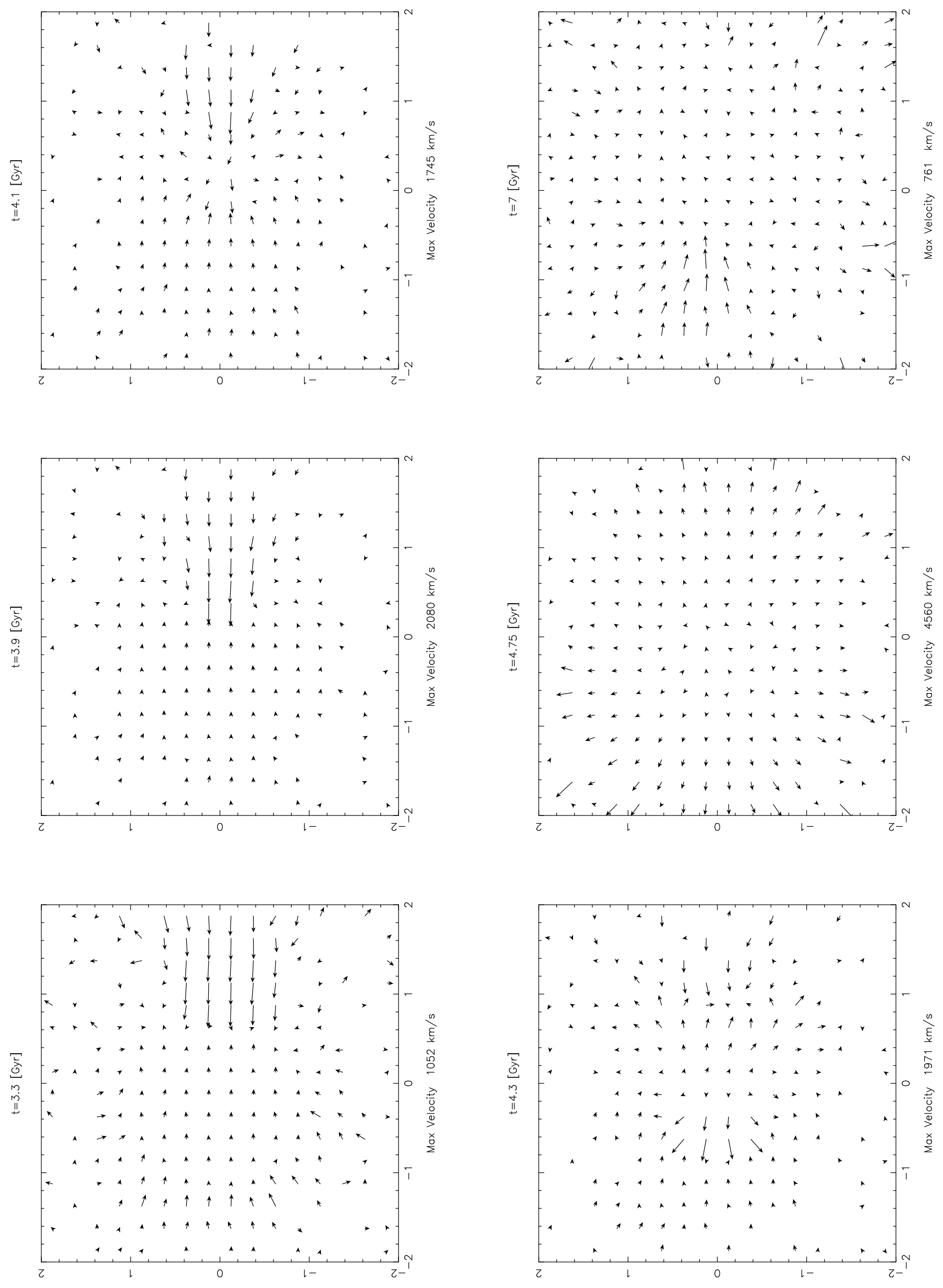

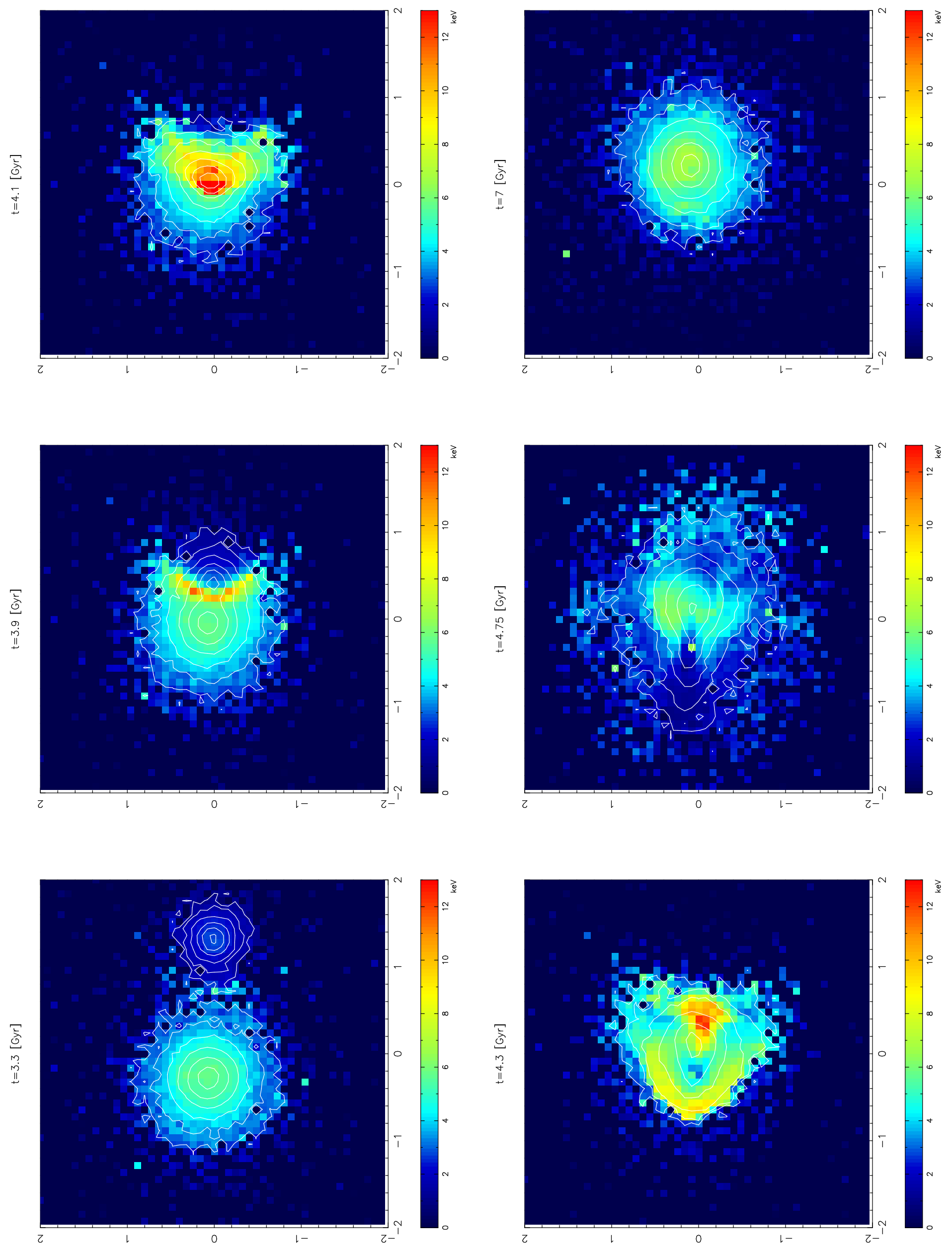

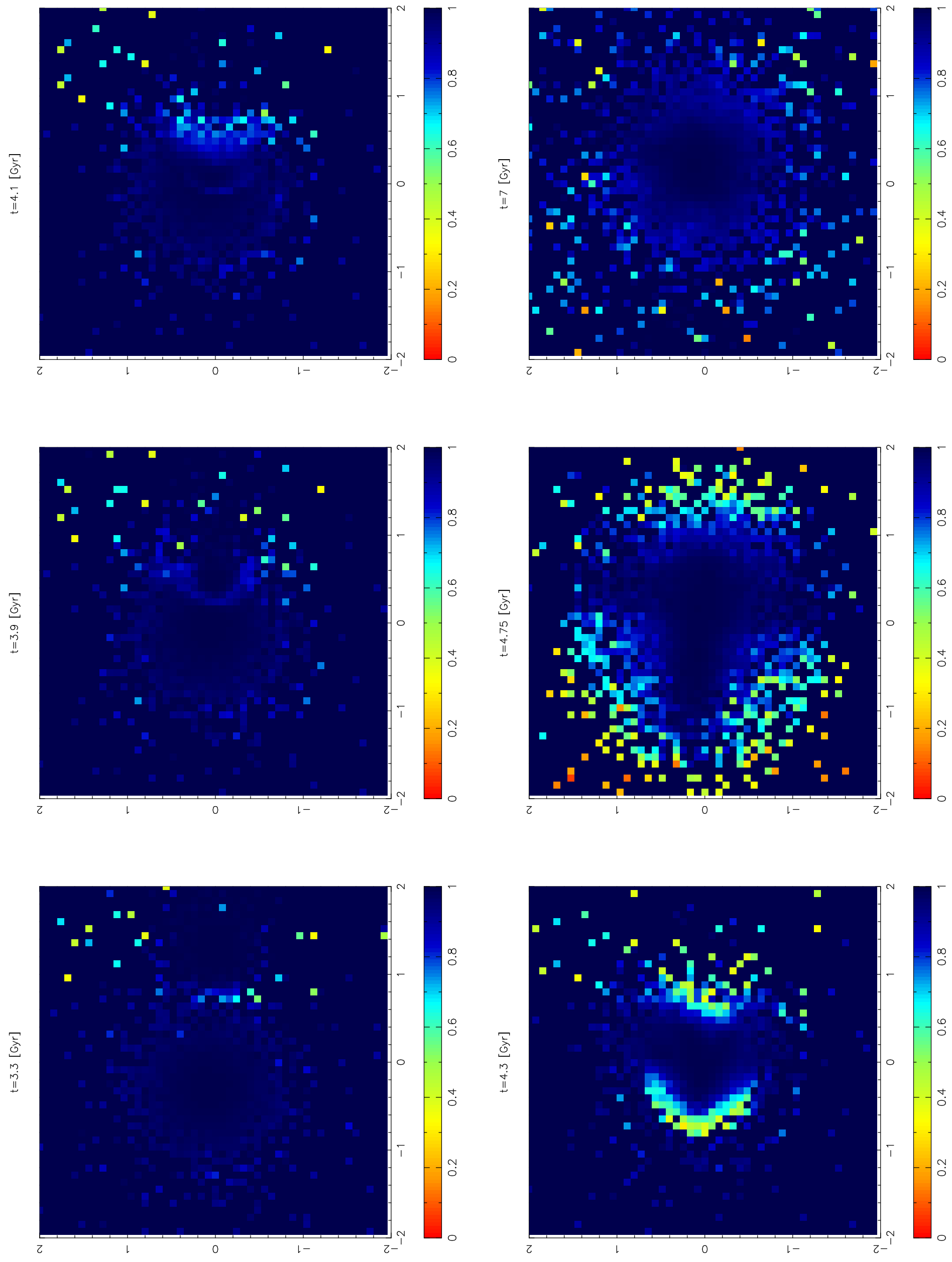